\title{
Optimal Intermittence in Search Strategies under Speed-Selective Target Detection
}

\author{
Daniel Campos, ${ }^{1}$ Vicenç Méndez, ${ }^{1}$ and Frederic Bartumeus ${ }^{2}$ \\ ${ }^{1}$ Grup de Física Estadística, Departament de Física. Facultat de Ciències, Universitat Autònoma de Barcelona, \\ 08193 Bellaterra (Barcelona) Spain \\ ${ }^{2}$ Centre d'Estudis Avançats de Blanes (CEAB-CSIC), Accés Cala Sant Francesc 14, 17300 Blanes, Girona, Spain
}

(Received 11 July 2011; published 10 January 2012)

\begin{abstract}
Random search theory has been previously explored for both continuous and intermittent scanning modes with full target detection capacity. Here we present a new class of random search problems in which a single searcher performs flights of random velocities, the detection probability when it passes over a target location being conditioned to the searcher speed. As a result, target detection involves an $\mathrm{N}$-passage process for which the mean search time is here analytically obtained through a renewal approximation. We apply the idea of speed-selective detection to random animal foraging since a fast movement is known to significantly degrade perception abilities in many animals. We show that speedselective detection naturally introduces an optimal level of behavioral intermittence in order to solve the compromise between fast relocations and target detection capability.
\end{abstract}

DOI: 10.1103/PhysRevLett.108.028102

PACS numbers: 87.10. $-\mathrm{e}, 02.50 .-\mathrm{r}, 05.40 . \mathrm{Fb}$

Consider a predator trying to detect a sparse set of immobile targets homogeneously distributed in space from which no other previous information is available. Formally, this can be studied as a first-passage problem in a disordered media. For such situations it has been shown both analytically and numerically [1-4] that Lévy flights represent an optimal search strategy since they minimize the mean time for detection or mean first-passage time. The alternation between small jumps in a given region (clustering) and relatively frequent long jumps typical of Lévy motion seems to represent the key to this optimality, even in those cases when the Lévy process is superposed to other types of motion [5].

Furthermore, intermittent search strategies have also received great attention during the last years [6]. These strategies are characterized by a distinction between scanning periods (diffusive movement) and relocation periods (ballistic movement) of random duration, so the target can only be detected during the scanning phases. Although the idea of intermittence is fundamental to understand many foraging or search processes, the drastic distinction made between scanning and relocation in previous models can be relaxed assuming that target detection is speed selective. In fact, intermittent search is based on the idea that in many animals fast movement degrades perceptual abilities $[7,8]$. Hence, the role of fast or long moves is to relocate the animal as fast as possible to new unexplored areas in prejudice of successful target detection. In the following, we address the question of what conditions make the incorporation of fast or long relocation moves advantageous in a search strategy. Under such conditions one may expect intermittence to arise evolutionarily.

Let us consider the idealized situation depicted in Fig. 1. The immobile targets are periodically located on a $1 \mathrm{D}$ domain at positions $\pm L, \pm 3 L, \pm 5 L, \ldots$ The predator starts from a random position $x_{0}$ in the interval $[-L, L]$ and moves by performing consecutive flights. The flight durations and velocities are assumed to be independent random variables distributed according to probability distribution functions (PDFs) $\varphi(t)$ and $h(v)$, respectively. Note that flight lengths and flight speeds are thus positively correlated. When the predator passes through a target location, detection occurs with probability $\Omega(v)$ and the target is missed otherwise. The function $\Omega(v)$ is assumed to be monotonically decreasing so the target detection gets more difficult as the predator moves faster.

Intermittence can be implemented in a natural and easy way within our speed-selective detection context. For instance, separating relocation and scanning could lead us to consider a bimodal distribution $h(v)$, with each peak in the distribution representing one of the two phases. Instead, we use here a different approach and define an intermittent search strategy as that where faster flights (at the risk of overpassing the target) and slower flights (to ensure target detection) are promoted, while intermediate speeds are

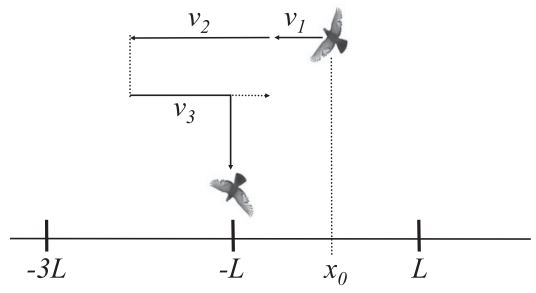

FIG. 1. Schematic representation of a search trajectory with speed-selective target detection. The individual performs flights with random velocities $v_{1}, v_{2}, v_{3}, \ldots$ and random durations. If it passes over a target location with a speed that is too large $\left(\boldsymbol{v}_{2}\right)$ the probability to miss the target is high; a later passage at a lower speed $v_{3}$ results in target detection. 
avoided in consequence. To illustrate the idea, let us consider a double exponential distribution (which is biologically sound $[9,10])$

$$
h(v)=\frac{1}{4 v_{0}}\left(\beta e^{-\beta|v| / v_{0}}+\frac{1}{\beta} e^{-|v| / \beta v_{0}}\right)
$$

where $0<\beta \leq 1$, and $v_{0}$ is an auxiliary parameter that will be taken equal to unity arbitrarily. Then for $\beta=1$ this becomes a single exponential distribution, and as $\beta$ decreases a larger fraction of faster flights (as well as slower flights) are allowed (see Supplemental Material [11]). So that, $\beta$ modulates the strength of intermittence (i.e., up to what extent intermediate speeds are avoided), with $\beta \rightarrow 0$ representing a highly intermittent strategy.

We start our analysis by showing (Fig. 2) how the mean search time to target detection $\langle T\rangle$ scales with the characteristic system size $L$. To this end we have carried out Monte Carlo simulations of the stochastic process depicted in Fig. 1. The results provided in Fig. 2 correspond for simplicity to the case $x_{0}=0$ and a fixed flight time $\varphi(t)=$ $\delta(t-\tau)$, with the speed flight distribution given by (1). Finally, we have considered a critical detection speed $v^{*}$, so $\Omega(v)=H\left(|v|-v^{*}\right)$ is a Heaviside function equal to 1 for flight speeds below $v^{*}$ and 0 otherwise.

As can be observed from the plot, for large $v^{*}$ the speedselective condition does not play any significant effect; then the detection time corresponds to the first-passage time and the results are almost trivial to interpret. In particular, for large $L$ the Wald's identity [12] from firstpassage processes is expected to hold; in our case this implies that $\lim _{L \rightarrow \infty}\langle T\rangle=L^{2} / \tau\left\langle v^{2}\right\rangle$. In Fig. 2 we have

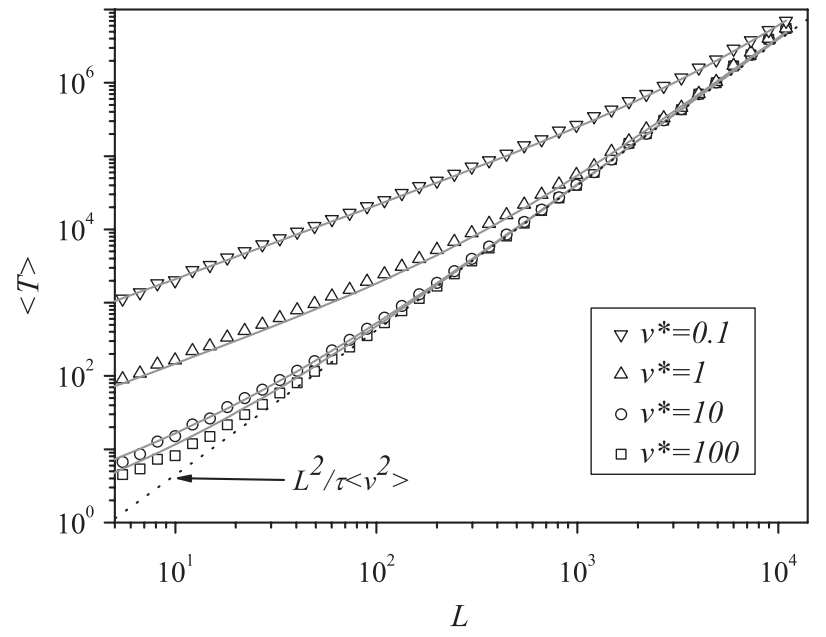

FIG. 2. Mean time required for target detection as a function of $L$. Different values of $v^{*}$ are considered (legend). Symbols represent results obtained from Monte Carlo simulations (averaged over $2 \times 10^{4}$ realizations) and solid lines correspond to the theoretical approximation (10). Dotted line represent the asymptotic theoretical results valid for first-passage processes. In all cases arbitrary values $\beta=0.2$ and $\tau=1$ have been used. plotted this asymptotic behavior (dotted line), which shows that the results for large $v^{*}$ values can be satisfactorily explained just in light of such approximation. However, as $v^{*}$ decreases and the problem departs from a first-passage process we observe a scaling $\langle T\rangle \sim L$ that persists even for relatively large values of $L$ (albeit the Wald's identity is finally fulfilled for $L$ large enough). Hence, in the following we derive an approximated method valid for the whole range of $v^{*}$ values.

Consider a particle performing flights of random durations and velocities, as in Fig. 1, but where the effects of target detection are not explicitly included. Since we are studying movement in a periodic domain we will consider equivalently that the particle moves in the finite domain $[-L, L]$ with periodic boundary conditions. We define $n_{1}\left(L, t ; x_{0}\right)$ as the flux of particles passing through the boundary $L$ (or $-L$ ) at time $t$ while moving with a speed small enough so that target detection would be possible. This can be written as

$$
\begin{aligned}
n_{1}\left(L, t ; x_{0}\right)= & \int_{0}^{\infty} v \Omega(v) p\left(L, v, t ; x_{0}\right) d v \\
& -\int_{-\infty}^{0} v \Omega(-v) p\left(-L, v, t ; x_{0}\right) d v
\end{aligned}
$$

where $p\left(x, v, t ; x_{0}\right)$ represents the probability density for the particles being at position $L$ at time $t$ moving with speed $v$. The first integral on the right-hand side of (2) accounts for those particles reaching the boundary $x=L$ from the left, while the second one stands for those reaching $x=-L$ from the right. Note that the distribution of first-detection times $f\left(L, t ; x_{0}\right)$ we want to find is not equal to $n_{1}\left(L, t ; x_{0}\right)$ since the latter includes all possible paths which have previously passed one, two, three,... times through $L$ or $-L$ slowly enough to allow target detection. So, we could subtract from $n_{1}\left(L, t ; x_{0}\right)$ the probability $n_{2}\left(L, t ; x_{0}\right)$ that at least two of these slow passages have occurred in the interval $(0, t)$ and the last one of these two occurs exactly at time $t$. Using an approach similar to that used in the Wilemski-Fixman approximation in chemical physics [13], this probability can be written as

$$
n_{2}\left(L, t ; x_{0}\right) \simeq n_{1}\left(L, t ; x_{0}\right) * n_{1}(L, t ; L)
$$

where the asterisk denotes time convolution. This is clearly a renewal approximation since we assume that, after the first target detection event, the motion process starts anew from $x_{0}=L$, neglecting any possible correlations. Note, however, that one will need to introduce as well a three detection function $n_{3}\left(L, t ; x_{0}\right)$, and so on. In the end, if we extend this discussion up to infinite order, we can approximate the first-detection time distribution (a more detailed derivation is provided in [11]) through

$$
f\left(L, t ; x_{0}\right) \simeq \sum_{i=0}^{\infty}(-1)^{i} n_{1}\left(L, t ; x_{0}\right) * n_{1}(L, t ; L)^{* i}
$$


where $f^{* i} \equiv f * \ldots * f$ is an $i$ th convolution product. After transforming from the time domain to the Laplace domain (with argument $s$ ) this takes the very simple form

$$
f\left(L, s ; x_{0}\right) \simeq \frac{n_{1}\left(L, s ; x_{0}\right)}{1+n_{1}(L, s ; L)} .
$$

We note that a similar derivation to that presented here was provided in [14] for the case of first-passage processes, while we have extended here the idea to our first-detection $(N$-passage) problem.

Hence, from (2) and (5) it follows that the problem of finding the first-detection time distribution reduces to finding a solution for $p\left(L, v, t ; x_{0}\right)$. This can be achieved from a continuous-time random walk formalism as follows. First, we introduce the probability density $j\left(x, t ; x_{0}\right)$ that a particle finishes a single flight (lands) at position $x$ at time $t$. Then

$$
\begin{aligned}
j\left(x, t ; x_{0}\right)= & j\left(x_{0}\right) \delta(t)+\int_{0}^{t} d \mathrm{\varsigma} \int_{-L}^{L} d z j\left(z, \mathrm{~s} ; x_{0}\right) \\
& \times \Psi(x-z, t-\mathrm{\varsigma})
\end{aligned}
$$

holds, with both $x$ and $z$ restricted to the finite domain $[-L, L]$. The first term in the right-hand side of (6) stands for the initial conditions, while the second term contains the contribution from all particles that fly from any position of the domain to $x$, with $\Psi(x, t)$ being the PDF that a single flight has length $x$ and duration $t$. The function $\Psi(x, t)$ is then related to the PDFs $\varphi(t)$ and $h(v)$ through [15-17]

$$
\Psi(x, t)=\int_{-\infty}^{\infty} d v \delta(x-v t) \varphi(t) h(v)
$$

The probability density $j(x, t)$ is connected to $p\left(x, v, t ; x_{0}\right)$ through

$$
p\left(x, v, t ; x_{0}\right)=\int_{0}^{t} d \varsigma j\left(x-v \varsigma, t-\varsigma, x_{0}\right) h(v) \int_{\varsigma}^{\infty} d \varsigma^{\prime} \varphi\left(\varsigma^{\prime}\right) .
$$

The expressions (6) and (8), defined just in $[-L, L]$, can be formally extended to $x \in \mathbb{R}$ by using periodic summation as shown in $[18,19]$. So we are able to find an exact solution for $p\left(x, v, s ; x_{0}\right)$ in the Laplace space as a Fourier series $\quad p\left(x, v, s ; x_{0}\right)=\sum_{m=-\infty}^{\infty} c_{m}\left(v, s, x_{0}\right) e^{-i \pi m x / L} \quad$ (see [11] for details). The exact expression of the Fourier coefficients reads

$$
c_{m}\left(v, s, x_{0}\right)=\frac{h(v) e^{-i \pi m x_{0} / L}\left[1-\varphi\left(s+\frac{i m \pi v}{L}\right)\right]}{2(L s+i m \pi v)\left[1-\hat{\Psi}\left(\frac{n \pi}{L}, s\right)\right]},
$$

where $\hat{\Psi}(k, s)$ represents the Fourier-Laplace transform of $\Psi(x, t)$. From this result, the general form of the firstdetection time distribution and its mean value can be computed analytically (see [11]). The result becomes specially simplified if we explore the limit $m \pi / L \ll 1$, which is equivalent to assuming that the Fourier series is basically dominated by the first $m$ modes; in principle this approximation is expected to work better as $L$ increases. Taking the case $x_{0}=0$ in concordance with the simulations presented in Fig. 2 we finally obtain

$$
\langle T\rangle=\frac{2 L}{\langle|v| \Omega(v)\rangle}+\frac{\langle t\rangle L^{2}}{\left\langle t^{2}\right\rangle\left\langle v^{2}\right\rangle},
$$

where $\langle t\rangle$ and $\left\langle t^{2}\right\rangle$ are the first and second order moments of the flight time distribution $\varphi(t)$, and $\left\langle v^{2}\right\rangle,\langle|v| \Omega(v)\rangle$ are averages over the speed distribution $h(v)$, so $\langle|v| \Omega(v)\rangle$ can be understood as the mean detection speed. Note that this result is completely independent of the explicit choice of $\varphi(t), h(v)$ and the detection probability $\Omega(v)$, provided that their first and second order moments converge.

In Fig. 2 we provide the corresponding results (solid lines) obtained from (10), which are in excellent agreement with the Monte Carlo simulations (even for rather small $L$, surprisingly). Not only can we reproduce the numerical results, but our approximation also allows us to justify the transition from $\langle T\rangle \sim L$ to $\langle T\rangle \sim L^{2}$. When the speedselective condition becomes very restrictive the first term in (10) increases and the linear scaling holds. Instead, if $\langle|v| \Omega(v)\rangle$ is large enough then the dominant term in (10) is the second one (specially as $L$ increases), which recovers the Wald's identity [note that for $\varphi(t)=\delta(t-\tau)$ this term becomes $\left.L^{2} / \tau\left\langle v^{2}\right\rangle\right]$.

We finally focus on the question of intermittence and the optimality of fast relocations. In Fig. 3(a) we plot the values of $\langle T\rangle$ as a function of $\beta$ and for different values of $v^{*}$, using the same distributions as in Fig. 2. It is clear that there exists an optimal level of intermittence $\beta$, specially for intermediate $v^{*}$ values (for $v^{*} \rightarrow 0$ and $v^{*} \rightarrow \infty$ the optimal $\beta$ tends asymptotically to 0 ). This is
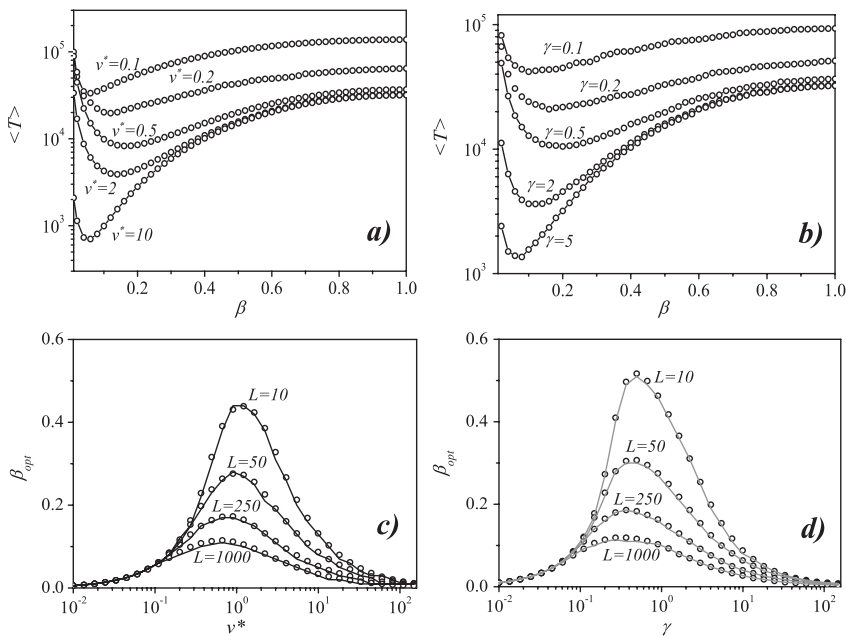

FIG. 3. Upper panels: Mean times to target detection as a function of $\beta$ for the speed distribution (1) with $\Omega(v)=$ $H\left(|v|-v^{*}\right)$ (left) and $\Omega(v)=e^{-|v| / \gamma}$ (right). Lower panels: Optimal values of the intermittence parameter $\beta$ as a function of $v^{*}$ and $\gamma$, respectively. Solid lines correspond to values obtained from the approximation (10). In all the cases, $\tau=1$. 
complemented by Fig. 3(c), where the optimal values of $\beta$ are plotted as a function of $v^{*}$ for different $L$. We observe that for larger $L$ the optimal level of intermittence tends to smaller $\beta$, since in that case strategies based on fast relocations get more benefit. We stress that different choices of $\varphi(t), h(v)$ and $\Omega(v)$ (including the Lévy case, which has also attracted attention within the context of intermittence [20]) have been explored, and the results are always qualitatively similar. For instance, we also show [Figs. 3(b) and 3(d)] the case $\Omega(v)=e^{-|v| / \gamma}$ to confirm that our results do not depend on the particular form of the detection probability. Actually, our expression (10) allows us to reach rather general conclusions regarding the question of optimality. For typical speed distributions found in tracking experiments with animals or microorganisms (e.g., exponential, Gaussian, Weibull,...) increasing the mean speed also involves increasing $\left\langle v^{2}\right\rangle$ but at the expense of reducing considerably $\langle|v| \Omega(v)\rangle$; on the other hand, very low mean speed results in small $\left\langle v^{2}\right\rangle$. So, from (10) both situations are detrimental, which means that necessarily a compromise between fast relocations and slow scanning must be reached, that is, an optimal level of intermittence. A different solution to this trade-off would be that searchers use a bimodal distribution (one slow mode and one fast mode, as in [6]); then it would be possible to increase $\left\langle v^{2}\right\rangle$ very much without reducing $\langle|v| \Omega(v)\rangle$. A relevant biological question would be what types of intermittence (e.g., bimodal strategies, suppression of intermediate speeds) would emerge under different evolutionary conditions.

In summary, we have explored both numerically and analytically a search problem in which target detection is conditioned to the searcher speed. We have proved that this plays a critical role in search efficiency. Under speedselective detection, the mean detection time shows a different scaling with the system size compared to the mean first-passage time: the convergence to $\langle T\rangle \sim L^{2}$ (Wald's identity) is much slower than in a first-passage problem, with a linear transient, i.e., $\langle T\rangle \sim L$ which has been analytically justified. Our approach can be extremely relevant in animal foraging where prey detection dynamics has been suggested to resemble more an $N$ passage rather than a first-passage search problem [5,21]. We also show that speed-selective detection imposes a trade-off between fast relocation to unexplored areas and target detection. The solution to this trade-off determines the optimal frequency of fast or long relocation moves that maximizes the search efficiency. This can naturally introduce an optimal level of behavioral intermittence as an evolutionary force in animal search strategies.

This research has been partially supported by Grants No. FIS 2009-13370-C02-01 (V. M.) and No. SGR
2009-00164 (V. M., D. C.). F. B. acknowledges the Spanish ministry of Science and Innovation (REF. 2009-04133, BFU2010-22337).

[1] G. M. Viswanathan, S. Buldyrev, S. Havlin, M. G. E. da Luz, E.P. Raposo, and H.E. Stanley, Nature (London) 381, 413 (1996).

[2] S. V. Buldyrev, S. Havlin, A. Ya. Kazakov, M. G.E. da Luz, E.P. Raposo, H.E. Stanley, and G.M. Viswanathan, Phys. Rev. E 64, 041108 (2001).

[3] F. Bartumeus, J. Catalan, U. L. Fulco, M. L. Lyra, and G. M. Viswanathan, Phys. Rev. Lett. 88, 097901 (2002); 89, 109902(E) (2002).

[4] R. Metzler, T. Koren, B. van den Broek, G. J. L. Wuite, and M. A. Lomholt, J. Phys. A 42, 434005 (2009).

[5] F. Bartumeus and S. A. Levin, Proc. Natl. Acad. Sci. U.S.A. 105, 19072 (2008).

[6] O. Bénichou, M. Coppey, M. Moreau, P.-H. Suet, and R. Voituriez, Phys. Rev. Lett. 94, 198101 (2005); C. Loverdo, O. Bénichou, M. Moreau, and R. Voituriez, Phys. Rev. E 80, 031146 (2009); O. Bénichou, C. Loverdo, M. Moreau, and R. Voituriez, Phys. Rev. E 74, 020102(R) (2006); Rev. Mod. Phys. 83, 81 (2011).

[7] W. J. Brien, H. I. Browman, and B. I. Evans, Am. Sci. 78, 152 (1990).

[8] D. L. Kramer and R. L. McLaughlin, American Zoologist 41, 137 (2001).

[9] H. Takagi, M. J. Sato, T. Yanagida, and M. Ueda, PLoS ONE 3, e2648 (2008).

[10] N. Srivastava, D. A. Clark, and A. D. T. Samuel, J. Neurophysiol. 102, 1172 (2009).

[11] See Supplemental Material at http://link.aps.org/ supplemental/10.1103/PhysRevLett.108.028102 for detailed calculations.

[12] S. Redner, A Guide to First-Passage Processes (Cambridge University Press, Cambridge, 2001).

[13] G. Wilemski and M. Fixman, J. Chem. Phys. 60, 866 (1974).

[14] T. Verechtchaguina, I. M. Sokolov, and L. SchimanskyGeier, Phys. Rev. E 73, 031108 (2006).

[15] V. Zaburdaev, M. Schmiedeberg, and H. Stark, Phys. Rev. E 78, 011119 (2008).

[16] M. Schmiedeberg, V. Y. Zaburdaev, and H. Stark, J. Stat. Mech. (2009) P12020.

[17] I. Gómez-Portillo, D. Campos, and V. Méndez, J. Stat. Mech. (2011) P02033.

[18] I. Calvo, B. A. Carreras, R. Sánchez, and B. Ph. van Milligen, J. Phys. A 40, 13511 (2007).

[19] B. Ph. van Milligen, I. Calvo, and R. Sánchez, J. Phys. A 41, 215004 (2008).

[20] M. A. Lomholt, T. Koren, R. Metzler, and J. Klafter, Proc. Natl. Acad. Sci. U.S.A. 105, 11055 (2008).

[21] A. M. Reynolds and F. Bartumeus, J. Theor. Biol. 260, 98 (2009). 